\title{
Opnet Based simulation for route redistribution in EIGRP, BGP and OSPF network protocols
}

\author{
Shalley Bakshi ${ }^{1}$, Ms. Suman ${ }^{2}$ \\ ${ }^{I}$ (Electronics and Communication, SSIET /PTU, India) \\ 2 ((Electronics and Communication, SSIET /PTU, India)
}

\begin{abstract}
Route redistribution (RR) has become an integral part of IP network design as the result of a growing need for disseminating certain routes across routing protocol boundaries. While RR is widely used and resembles BGP in several non-trivial aspects, surprisingly, the safety of $R R$ has not been systematically studied much by the networking community. This paper presents the study of the model in opnet for understanding the route redistribution in a network comprising of different AS. The behaviour of routing protocols EIGRP, OSPF, $B G P$ is studied. The performance of each routing protocol is different from each other. Each of them has different architecture, adaptability, routing algorithms, processing delays and convergence capabilities. Among different routing protocols, EIGRP and OSPF have been considered as the pre-eminent routing protocols for the real -time application. To select a right protocol, several parameters such as network convergence time, bandwidth, scalability are considered. This paper reports a simulation based study between EIGRP, BGP and OSPF. In order to evaluate the performance of EIGRP and BGP three network scenarios are configured viz route redistribution, $F F C$ with route redistribution and to fasten the BGP process the header changes are done.
\end{abstract} Keywords: EIGRP, BGP, OPNET, Load Balancing, Routing Updates, Autonomous System, Routing Policy.

\section{INTRODUCTION}

The internet is made of tens of thousands of different networks called autonomous systems (AS),and $\mathrm{BGP}$ is used to communicate reachability information .Moreover working over large scale IP networks ,moving between different sub-networks and reach the final destinations, is almost impracticable without using a routing protocol. The routing protocols are found in network layer. The main aim of the network layer is to support the appropriate path from origin to destination, even though both are not connected directly. This layer is responsible for establishing, maintaining and terminating the connection. Also, this level defines the routing and controls the congestion packets in a sub-network. Devices that are assigned to this task are called routers. A router is a device that works at layer3 which is responsible for sending datagrams from one network to another network directly connected, and it is in charge of interpreting the routing protocols to carry information from origin to destination. There is more than one routing protocol used in different ASes so in order to enhance the communication between different sub-networks concept of route redistribution is introduced [1]. In multihoming environment, if a link fails in the AS, it can still connect to the Internet via other connections. The reliability of the Internet depends upon the reaction time necessary for the underlying routing protocols finding the backup paths in case of failures. There are networks which show that BGP fails to take advantage of redundant connection and implementing fault tolerant configuration cannot ensure continuous connectivity when primary connection fails. The end user of a network does not know how data flows between the devices [2]. The user desires to receive the information as soon as possible, no matter on what happens on the network. Moreover, the received information should be complete without losses generated by network failures. These features are known as the fault tolerance of a network. These issues are resolved by the route redistribution which not only interconnects routing protocols but strengthens the reliability of inter-domain routing. The routing algorithm is responsible for determining a possible alternative route in case of a link failure, or other topology changes.

\section{RELIABILITY CHALLENGES}

In multi-homing environment if a link fails in the AS, it can still connect to the Internet via other connections. The reliability of the Internet depends upon the reaction time necessary for the underlying routing protocols finding the backup paths in case of failures. There are networks which show that BGP fails to take advantage of redundant connection and implementing fault tolerant configuration cannot ensure continuous connectivity when primary connection fails. The end user of a network does not know how data flows between the devices. The user desires to receive the information as soon as possible, no matter on what happens on the network. Moreover, the received information should be complete, without losses generated by network failures [3]. These features are known as the fault tolerance of a network. These issues are resolved by the route redistribution which not only interconnects routing protocols but strengthens the reliability of inter-domain 
routing. The routing algorithm is responsible for determining a possible alternative route in case of a link failure or other topology changes.

\section{FAST FAILOVER CONFIGURATION}

\section{ROUTE REDISTRIBUTION}

Different routing protocols can be configured in a single router. BGP is used to determine routing information between Autonomous Systems, while an Interior Gateway protocol (IGP) is used to resolve routes within an AS. In order to allow different routing protocols to exchange routing information, route redistribution can be used. A router running multiple routing processes does not by default redistribute routes among these processes[4]. RR must be explicitly configured.

\section{BASIC IDEA}

Fast Failover Configuration (FFC) uses the IGP to convey BGP routes. FFC uses BGP to advertise the backup routes and in particular IGP to carry primary routes by route redistribution. During redistribution each routing protocol keeps the separate routing table and uses different route decision algorithms in order to select the best route. In the realm of interior routing protocols, numerous studies have been published about the behavior of OSPF and EIGRP. These studies have contributed with a lot of potential insights of interior routing protocols (e.g. EIGRP and OSPF)[5], which has drawn similar attention to work in that direction.

\section{IMPLEMENTATION}

This work is divided into three scenarios. First scenario involves the analysis of the various routing protocols like EIGRP, OSPF and BGP. This analysis has been done with route redistribution with EIGRP as a reference protocol. We assume that all routers are running both BGP and IGP and use Cisco's Ad values to determine their forwarding routes. In FFC, BGP is the backup backbone protocol while EIGRP, OSPF are chosen to be the primary backbone protocols. All the routers are running both the IGP and BGP[6].

Routing Protocols become one of the important decisions in the design of the network. An important aspect of routing protocol is how quickly it converges when there is a change in the topology table.[7] Convergence is when the routing tables of all routers have complete and accurate information about the network. Convergence time is the time it takes routers to share information, calculate best path and update their routing tables. Routing protocols that converge with in the minimum amount of time are considered to be efficient. The convergence time is emphasized in this scenario.

\section{SCENARIO IMPLEMENTATION IN OPNET}

EIGRP can be configured in the network model by either router configuration import or manually. The typical node model architecture of EIGRP in OPNET is shown in the fig.1. EIGRP uses the DUAL (Diffusion Update Algorithm).All route calculations are done by the DUAL

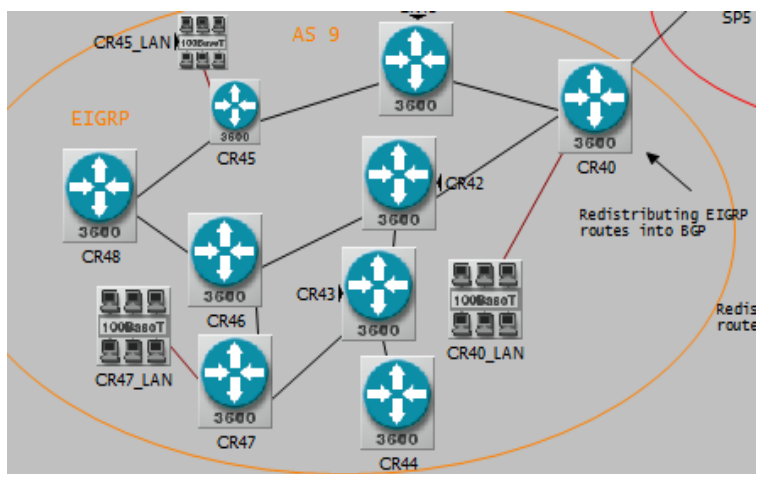

Fig.1.Customer Network with EIGRP

EIGRP scales well and converges quickly with minimal network traffic. To minimize its load on the network, EIGRP propagates only routing table changes instead of the entire routing table when a change occurs. This scenario contains one of the AS configured with EIGRP. 


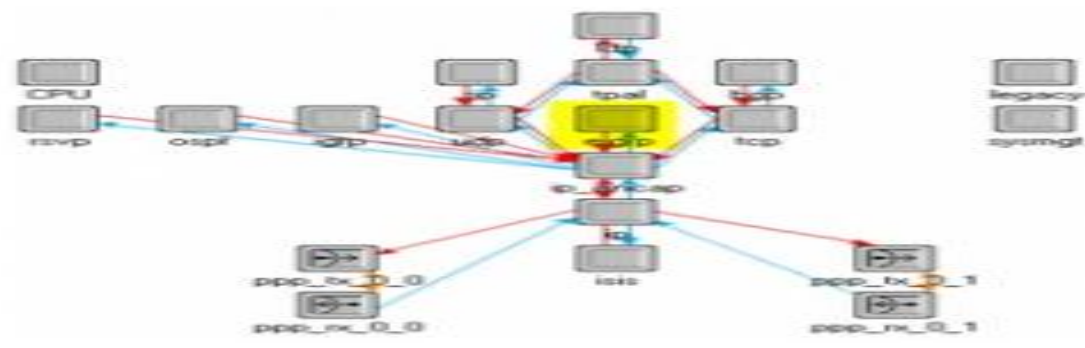

Fig.2.Node Model

The architecture of the EIGRP process model (Fig.3) is designed based on the tasks defined in the EIGRP. There are two process models EIGRP as parent process and EIGRP as a child process for each AS process [8].

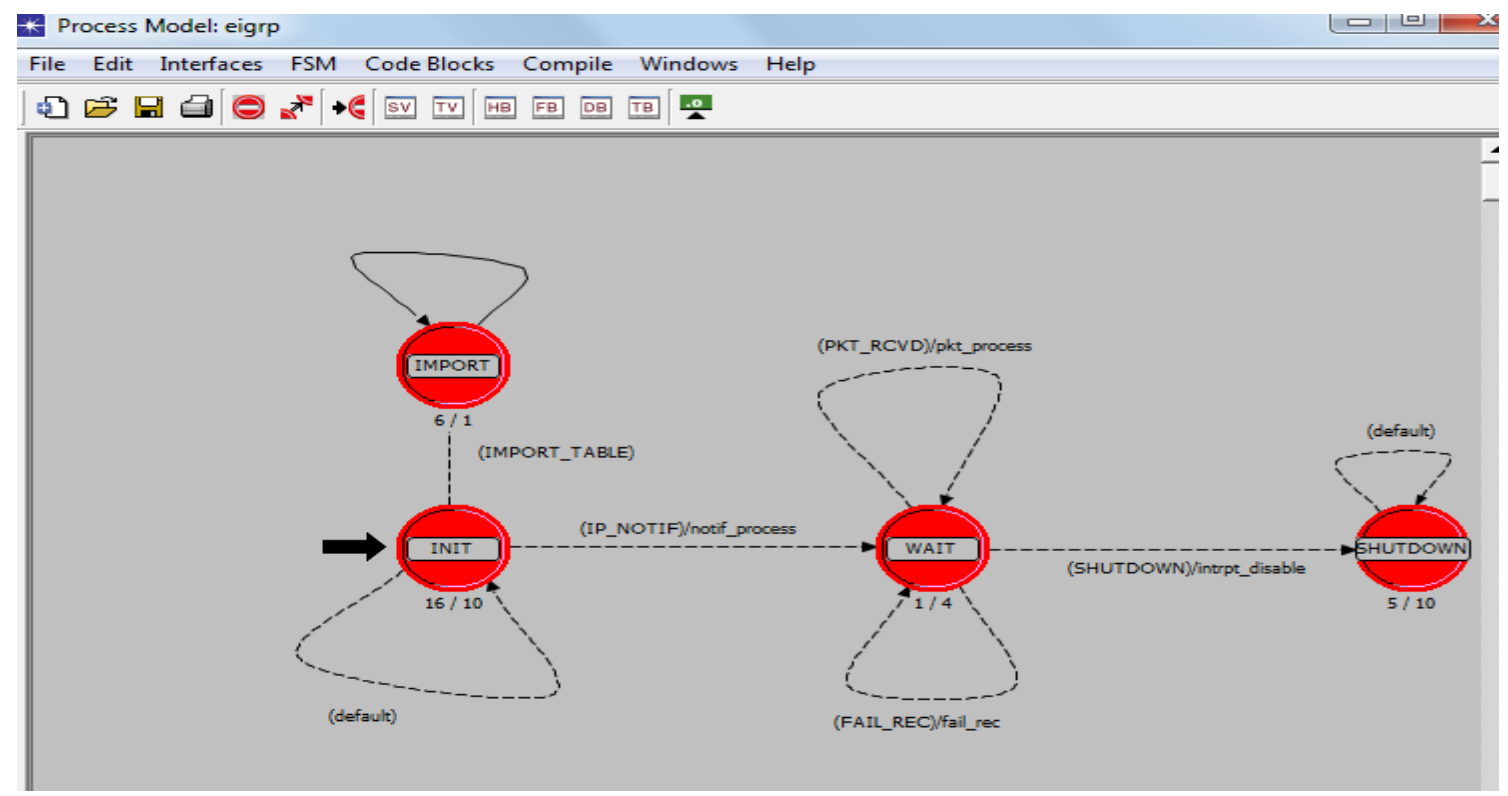

Fig.3.Process Model

In case of topology changes, the EIGRP process tries to recover quickly using topology table to find an alternate route if not so, the EIGRP process starts DUAL, which queries neighbor nodes topology table for an alternate route. Thus there is an exchange of update messages. The EIGRP network reaches convergence when there are no active query, reply or update messages in the network.

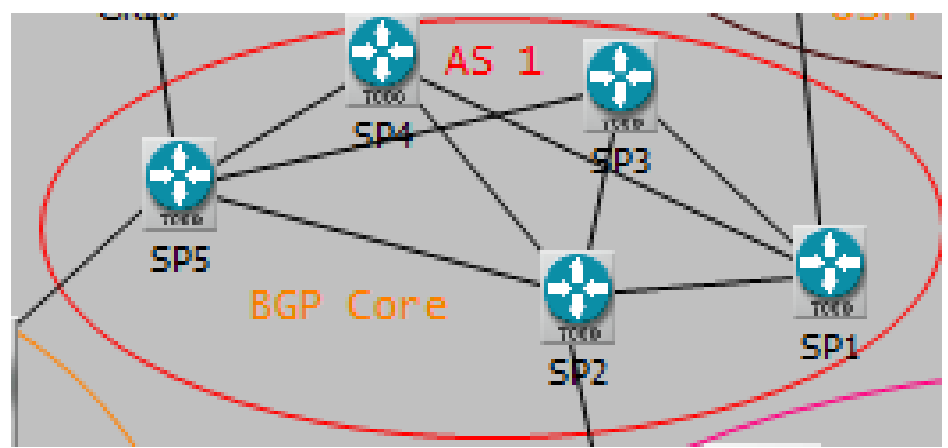

Fig.4. BGP core Network

In second scenario, Fast failover configuration is implemented where in the node architecture of the BGP routers is updated. Each router in AS with BGP has a header set to 1.[9] If a router receives the route from iIGP or iBGP route to the same destination, the router chooses IGP-derived routes because the AD Of IGP is always less then $\mathrm{AD}$ of $\mathrm{iBGP}(200)$.Router adds the IGP route to its forwarding table .In case IGP the route fails ,the router uses the $\mathrm{iBGP}$ derived routing information. The impact of $\mathrm{AD}$ is not seen in this situation. Each 
backbone router in the AS with BGP has two available BGP routes and one IGP route. The convergence duration and the traffic sent and received is analyzed in OPNET [10].

As BGP is complex, to understand/configure and very slow to converge so in our technique, instead of comparing $\mathrm{AD}$ value and making IGP primary and i-BGP secondary we will run i-bgp on all the routers and make it primary. We work on the update timers of BGP so as to decrease the downtime [5]. For this, header changes have been done to fasten the routing updates. It is easy to work on the earlier approach in case of link failure i.e when the best path fail then it does not convey BGP routes on IGP routes that is by comparing AD value of both protocols. Instead of this we work on the BGP update timers to decrease the time of updates to as to increase the process speed that will help us in case of link failure.

\section{RESULTS AND DisCUSSIONS}

Simulations have revealed the importance of route redistribution and the behaviour of EIGRP, BGP in detail. For experimentation, we have used OPNET simulator 14.05 with logical area of $10 \mathrm{~km} \times 10 \mathrm{~km}$ work area with three autonomous systems. Heavy duty routers are used with high data rate links in between autonomous systems so that communication is effective.

\section{A. FFC BGP Network Convergence duration}

FFC uses BGP to advertise the backup routes, and IGP to carry the primary route by route redistribution. When there is redistribution between routing protocols, each routing protocol keeps the same routing information at a separate routing table, and uses different route decision algorithms to select the best route.

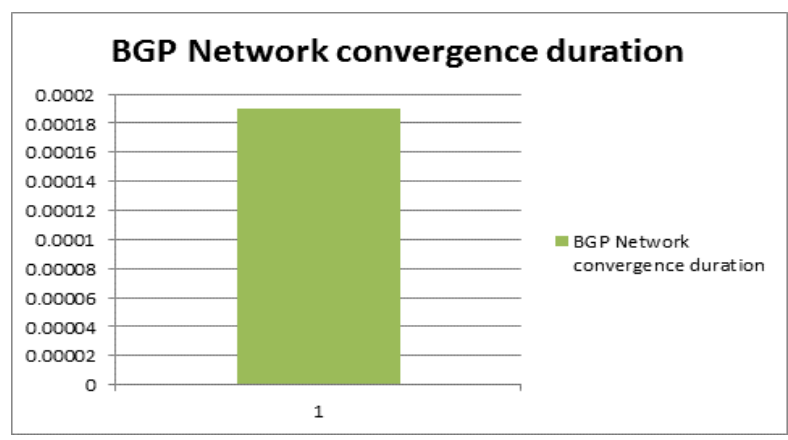

Fig.5. BGP Network Convergence Duration

\section{B. FFC BGP Packet loss}

It has been found the duration of packets loss after some intervals comes out to be approximately equal to 4 in case of FFC network topology.

\begin{tabular}{|c|c|c|}
\hline $\begin{array}{c}\text { FFC: } \\
\text { BGP Traffic } \\
\text { Received } \\
\text { (bits/sec) }\end{array}$ & $\begin{array}{c}\text { FFC: } \\
\text { BGP Traffic } \\
\begin{array}{c}\text { Sent } \\
\text { (bits/sec) }\end{array}\end{array}$ & $\begin{array}{c}\text { Packets } \\
\text { loss } \\
\text { (bits/sec) }\end{array}$ \\
\hline 24.75 & 28.6 & 3.85 \\
\hline
\end{tabular}

Fig.6. Packet Loss

\section{Proposed BGP Convergence}

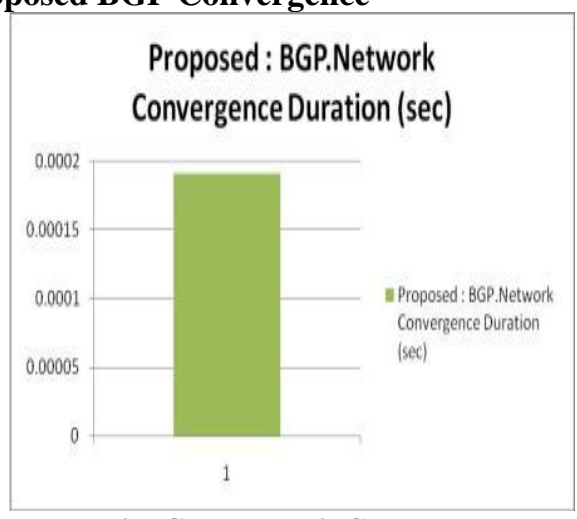

Fig.7. Proposed BGP Network Convergence Duration

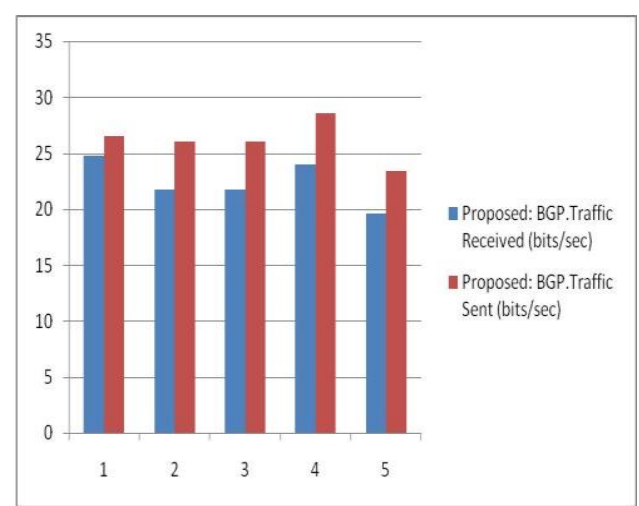

Fig.8. Proposed BGP packet loss 


\section{FFC EIGRP Network convergence duration}

The convergence time for EIGRP in seconds comes out to be 0.000408 . So this is clear from this that the convergence duration of EIGRP in the FFC scenario is same as the convergence duration of EIGRP in the base paper implementation.

\section{E. FFC Packet loss EIGRP}

By improving BGP Duration of Packet Loss in proposed work we have also managed the duration of Packet Loss of EIGRP in our FFC work to be as same that of base paper.

\section{F. Proposed convergence in EIGRP}

Fig.9 shows the value of EIGRP convergence time as the simulation time increases. So at times that is shown in the values 5.000449 and 70.00301 the convergence duration that is in seconds comes out to be 0.000408 and 0.002468 .

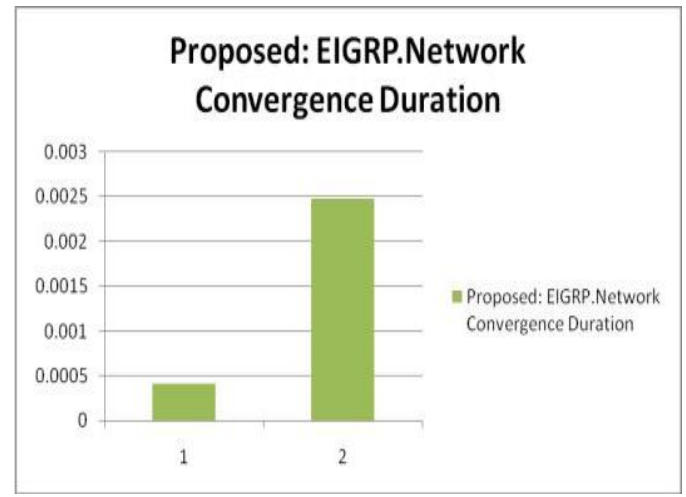

Fig.9.Proposed EIGRP Network convergence duration

So this is clear that the convergence duration of EIGRP in the proposed work is same as the convergence ration of EIGRP in the base paper implementation. So by improving BGP convergence duration in proposed work we manage the convergence duration of EIGRP in our proposed work to be as same that of base paper.

\section{G.Proposed duration of packet loss in EIGRP}

So the duration of packets loss is 151.47.So this is clear from this simulation that the Duration of Packet Loss of EIGRP in the proposed work is same as the Duration of Packet Loss of EIGRP in the base paper implementation. So by improving BGP Duration of Packet Loss in proposed work we have also managed the duration of Packet Loss of EIGRP in our proposed work to be as same that of base paper.

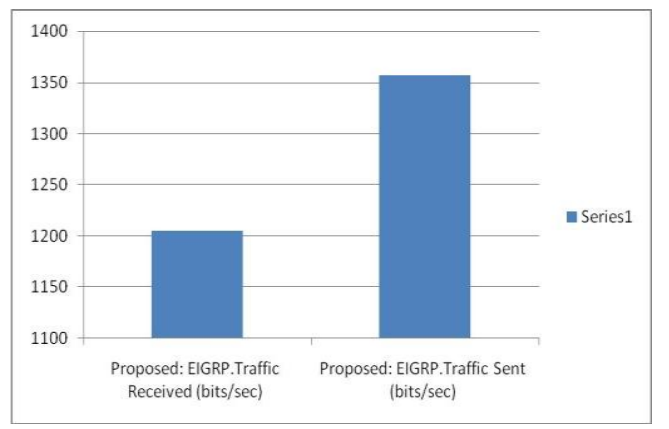

Fig.10.Proposed duration of packet loss in EIGRP

\section{CONCLUSION}

Performance has been evaluated on the basis of various parameters aimed to figure out the effects of routing protocols. By comparing these protocols performances, we have come across that the EIGRP protocol performs better in terms of convergence duration packet loss. A detailed simulation study to the problem discussed in the simulation with the redistribution environment in OPNET helped to find the better solution. EIGRP can learn the topology information and updates more rapidly. 


\section{REFERENCES}

[1] Zhan-Zhen Wei, Feng Wang," Achieving Resilient Routing through Redistributing Routing Protocols", Communications (ICC), IEEE International Conference, pp 1-5, 2011

[2] Kevin Butler, Patrick McDaniel," A Survey of BGP Security Issues and Solutions", Proceedings of the IEEE, Volume-98, No- 1, January 2010

[3] R. Perlman, Interconnections," Bridges, Routers, Switches, and Internetworking Protocols”, 2nd ed. Reading, MA: Addison Wesley, 1999.

[4] C. Ellison and B. Schneier," BTen risks of PKI: What you're not being told about public key infrastructure", Comput. Security J., vol. 16, no. 1, 2000 .

[5] M. Lepinski and S. Kent," An Infrastructure to Support Secure Internet Routing", Internet Draft draft-ietf-sidr-arch-08.txt, Jul. 2009.

[6] Thomas C. Bressoud, Rajeev Rastogi," Optimal Configuration for BGP Route Selection", INFOCOM 2003. Twenty-Second Annual Joint Conference of the IEEE Computer and Communications. IEEE Societies, vol. 2, pp 916- 926, 2003.

[7] Bin Wang," The Research of BGP Convergence Time", Information Technology and Artificial Intelligence Conference (ITAIC), 6th IEEE Joint International Conference, vol- 2, pp 354-357, 2011.

[8] Jong Han Park, Ricardo Oliveira, Shane Amante," BGP Route Reflection Revisited" , IEEE Communications Magazine, Vol- 50, Issue- 7, pp 70-75, July 2012.

[9] Xiaozhe Zhang, Xicheng Lu, Jinshu Su, Baosheng Wang," SDBGP: A Scalable Distributed BGP Routing Protocol Implementation", High Performance Switching and Routing (HPSR), IEEE 12th International Conference, pp 191-196, 2011.

[10] Jaeyoung Choi, Jong Han Park, Pei-chun Cheng, Dorian Kim," Understanding BGP Next-hop Diversity", IEEE Conference of Computer Communications Workshops, Vol.1, pp.846-851, 2011. 